\title{
Conference Paper \\ Cardiovascular Biomarkers in ACS: State of the Art 2012
}

\author{
Evangelos Giannitsis and Hugo A. Katus \\ Department of Internal Medicine III, Cardiology, University Hospital Heidelberg, 69120 Heidelberg, Germany
}

Correspondence should be addressed to Evangelos Giannitsis; evangelos_giannitsis@med.uni-heidelberg.de

Received 15 January 2013; Accepted 1 April 2013

Academic Editors: A. Bellou, C. Hamm, M. Möckel, and J. Searle

This Conference Paper is based on a presentation given by Evangelos Giannitsis at "Clinical Decisions in Acute Patients: ACSPOCT-Hypertension and Biomarkers" held from 19 October 2012 to 20 October 2012 in Berlin, Germany.

Copyright (C) 2013 E. Giannitsis and H. A. Katus. This is an open access article distributed under the Creative Commons Attribution License, which permits unrestricted use, distribution, and reproduction in any medium, provided the original work is properly cited.

In the setting of an acute coronary syndrome cardiac troponins are well established for the diagnosis of myocardial infarction. In particular, diagnostic protocols using high-sensitivity troponin assays are being recommended for earlier diagnosis of MI by the European Society of Cardiology (ESC) guidelines on the management of ACS without ST segment elevation. In addition to accurate detection of myocardial necrosis, cardiac troponins give complementary information on short- and long-term prognosis and facilitate the identification of patients who derive benefits from a more aggressive anticoagulation and/or early invasive therapy versus conservative therapy. Other cardiac biomarkers may help to improve earlier diagnosis or improve risk stratification. Their role is currently under investigation. The present state-of-the-art paper gives an overview on the role of cardiac troponins including recent recommendations on the use of high-sensitivity assays from the third version of the Joint ESC/ACCF/AHA/WHF infarct definition "Universal MI definition" and the ESC guidelines. In addition, an overview on the role of novel cardiac biomarkers in earlier diagnosis or risk stratification is provided.

\section{Introduction}

Coronary artery disease is one of the leading causes of death worldwide [1]. Better understanding of the pathomechanism of disease, use of more potent dugs, and improved diagnosis have helped to reduce $\mathrm{CV}$ mortality although total numbers of MI increase. Nowadays cardiac troponins are the preferred biomarkers for detection of myocardial cell necrosis and are essential for the diagnosis of myocardial infarction $[2,3]$. The revised ESC/ACC definition of MI that had replaced the WHO definition of MI in the year 2000 [4] is now available in its third updated version [5]. This definition is supported by all major cardiology societies and by the WHO referred to as the third Universal MI definition. More recently, novel highly sensitive cTn assays are being increasingly used in clinical practice due to their superior analytical sensitivity allowing the detection of minute myocardial damage $[6,7]$. Current ESC guidelines endorse earlier diagnostic algorithms using such hsTn assays [8]. However, increasing rates of patients with detectable cTn levels, not due to an ACS, also started to confuse clinicians [9]. This paper gives an overview on the present role of cTn and hsTn assays in diagnosis, risk stratification, and guidance of therapy and provide a state-ofthe-art overview on new promising biomarkers for earlier or rapid rule-out of MI and for prognostic assessment.

\section{Universal MI Definition and Cardiac Troponin}

Cardiac biomarkers play a central role for the diagnosis and management of patients with an ACS. It is well established that cardiac troponins are the preferred biomarkers for diagnosis of myocardial infarction due to their absolute cardiospecificity and due to their ability to detect minute cardiomyocyte necrosis $[2,3]$. Accordingly, cardiac troponins have been implemented in contemporary definitions of MI $[4,5]$. The third version of the redefinition of MI was published in the year 2012 [5]. In this updated version, cardiac troponin continues to be the preferred biomarker of myocardial necrosis. An elevation of cTn exceeding the 99th percentile of a healthy reference population is diagnostic for an MI, provided that cTn shows a rise and/or a fall signaling an acute rather than a chronic elevation and only in the presence of myocardial ischemia. 
Criteria for MI (3rd Version of the Joint ESC/ACCF/AHA/ WHF Infarct Definition). The term acute myocardial infarction (MI) should be used when there is evidence of myocardial necrosis in a clinical setting consistent with acute myocardial ischemia. Under these conditions any one of the following criteria meets the diagnosis for MI (adapted from [5]).

Detection of a rise and/or a fall of cardiac biomarker values (preferably cardiac troponin (cTn)) with at least one value above the 99th percentile upper reference limit (URL) and with at least one of the following:

(i) symptoms of ischemia,

(ii) new or presumed new significant ST-segment-T wave (ST-T) changes or new left bundle branch block (LBBB),

(iii) development of pathological $\mathrm{Q}$ waves in the ECG,

(iv) imaging evidence of new loss of viable myocardium or new regional wall motion abnormality,

(v) identification of an intracoronary thrombus by angiography or autopsy.

As compared to the second version of the Universal MI definition, the criteria for a context of myocardial ischemia have been extended. Now detection of an intracoronary thrombus on coronary angiography or autopsy has been added to the list of criteria that includes symptoms, ECG changes, new wall motion abnormalities, or detection of loss of viable myocardium. In addition, 5 subtypes of MI have been defined according to their putative pathomechanism [5]. Strict adherence to the diagnostic criteria of the third version of the MI definition ensures a proper interpretation of troponin results given that troponin is due to myocardial cell necrosis but not obligatory due to myocardial ischemia [10]. The list of conditions that may cause an acute or chronic elevation of troponin in the absence of myocardial infarction is seemingly endless [10]. Discrimination of a type II MI, that is, characterized by myocardial ischemia arising from an imbalance of oxygen demand and supply from myocardial injury unrelated to the myocardial ischemia, is difficult as myocardial ischemia may be involved in some cases, and the definite reason for myocardial injury remains illusive in many conditions.

\section{High-Sensitivity Troponin Assays}

In an attempt to improve precision of conventional cTn assays at the lower detection range, manufacturers refined their assays that are now able to detect more than 2-fold lower troponin concentrations with an acceptable imprecision of less than $10 \%$ at the 99th percentile [11]. Now, hsTn assays can measure cardiac troponin in the majority of healthy individuals [12]. Improved analytical sensitivity of cTn also enabled earlier diagnosis of MI and detection of more infarcts at the cost of a declining number of unstable angina [13]. Based on findings from large clinical trials, ESC guidelines for management of NSTE-ACS promote the use of hsTn assays and recommend the implementation of an earlier rule-in and rule-out algorithm [8]. Accordingly, patients presenting with suspected ACS without ST-segment elevation need a cTn measurement on presentation. If cTn is still below the 99th percentile, retesting after 3 or 6 hours is recommended unless the time from the onset of symptoms to the presentation is longer than 6 hours [14]. An increase of cTn above the 99th percentile with a percent change of $50 \%$ or more qualifies for a diagnosis of NSTEMI. Smaller percent changes may signal a relevant differential diagnosis. It is anticipated that the majority of patients with suspected ACS will present with a baseline elevation of cTn [7]. Rule-in of NSTEMI requires repeat measurement of $c$ Tn to discriminate between a chronic and an acute change. By arbitrary convention, a change in either the direction of $20 \%$ or more serves as the kinetic criterion [14]. Patients with a very high cTn level on presentation have a high pretest probability of NSTEMI as a differential diagnosis is less likely [8]. These patients should undergo coronary angiography without an obligatory need to retest after 3 or 6 hours.

\section{Earlier Detection of MI}

One of the most attractive applications of hsTn assays is earlier rule-out of MI. While earlier guidelines recommended to repeat troponin testing 6 to 9 hours after presentation, the new algorithm recommends a repeat testing after 3 hours and optionally after 6 hours [8]. Study findings suggest that other earlier markers of ischemia such as myoglobin or heart type FABP do not provide additional information and thus may be obviated [15]. There is also emerging evidence that ruleout of an MI can be accomplished safely within 1 or 2 hours using a hsTn assay $[16,17]$. Such protocols would accelerate diagnostic workup, reduce observation times in overcrowded chest pain units or EDs, and allow a cost-effective and sage discharge of patients and avoid unnecessary hospitalization. However, such algorithms need to be validated prospectively in broad unselected patient cohorts with suspected ACS [18].

\section{Other Promising Biomarkers for Early Diagnosis}

There is accumulating evidence indicating that copeptin, the stable fragment of vasopressin, can improve rapid rule-out of MI when measured together with a conventional $[19,20]$ or a hsTn assay [21]. Following an index event such as a small infarct copeptin is rapidly released from the hypophysis and will be detectable in blood in patients with a later rise of cTn. Patients with a negative copeptin and cTn on presentation are unlikely to develop a NSTEMI at serial testing [19]. The performance of copeptin is excellent in combination with a conventional cTnT or cTnI assay where negative predictive value is $>99 \%[19,20]$. The added benefit of copeptin to a hsTn assay is smaller but still significant [21].

\section{Prognosis}

An obstacle with hsTn assays is that the higher sensitivity results in a substantially higher rate of patients with 
analytically true positive troponin results not due to ACS reflecting acute or chronic myocardial injury [22]. It may be challenging to discriminate between NSTEMI and noncoronary cardiac or extracardiac reasons of elevated cTn. Nevertheless, an elevated cTn is associated with an adverse outcome regardless whether the underlying reason is NSTEMI or nonACS [23]. The reasons for troponin elevations in the absence of myocardial ischemia are not fully understood but may be associated with a higher burden of atherosclerosis [24], more complex coronary lesions [25], depressed LV function, associated cardiac comorbidities, and severely impaired renal function [26]. Thus, elevated cTn may represent a surrogate parameter integrating prognostic informations from associated diseases.

Instead of labeling an elevated cTn in the absence of MI as a "false positive" finding one should rather search for the underlying etiology in order to provide an early and specific therapy.

The use of high-sensitivity cTn assays has further improved risk stratification by detecting patients at risk who were previously not detected by conventional cTn assays including small MIs [27] and patients in earlier stages of chronic pulmonary hypertension [28] or acute and chronic heart failure [29].

In ACS several other biomarkers have been identified to provide independent and additive prognostic information to $\mathrm{cTn}$. These biomarkers included markers of inflammation such as CRP, IL-6, and fibrinogen, markers of hemodynamic stress or volume overload, that is, natriuretic peptides or MR-proadrenomedullin [30,31], and indicators of impaired renal function such as creatinine, estimated GFR, or cystatin [32]. Little is known about the added value of these biomarkers when hsTn is used instead of conventional cTn. Recently, the utility of 14 novel biomarkers for prognostic assessment in ACS patients with undetectable conventional cTn level was investigated [33]. Along with hsTn an independent prognostic information was conferred only by GDF-15 and MR-proadrenomedullin. In another study, copeptin not only improved rapid rule-out of emerging NSTEMI when copeptin was low but also helped to identify patients at higher risk for adverse outcomes if copeptin levels were high [34].

Use of additional biomarkers or a panel of biomarkers appears attractive for the better understanding of the pathophysiological process behind ACS or other acute diseases and for a refined risk stratification (Figure 1). However, current guidelines preclude any recommendations for routine use because the incremental value over highly sensitive troponin tests has not been evaluated yet [8].

\section{Guidance of Therapy}

Cardiac troponins have been shown to help in the identification of patients who might benefit from more aggressive antithrombotic or antiplatelet therapies and derive more benefits from an early invasive strategy. For the former, cTn is regarded as a surrogate of a vulnerable or ruptured plaque with subsequent activation of platelets and clot formation. Accordingly, benefits of low molecular weight heparin [35, 36], GP IIb/IIIa inhibitors [37], and triple antiplatelet
Biomarkers for diagnosis and risk stratification in ACS

$$
\begin{aligned}
& \begin{aligned}
\text { Prognosis } & \text { BNP/NT-proBNP } \\
& (\mathrm{hs}) \text {-CRP }
\end{aligned} \\
& \text { Diagnosis }\left\{\begin{array}{l}
\text { Inflammation or oxidative stress: } \\
=\text { MPO } \\
\text { GDF-15 } \\
\text { LPAP-2A } \\
\text { Earlier ischemia markers: } \\
\text { hFABP } \\
\text { IMA } \\
\text { Copeptin }
\end{array}\right.
\end{aligned}
$$

"However, the incremental value-particularly over highly sensitive troponin tests-has not been evaluated, thereby presently precluding any recommendations for routine use"

ESC guidelines 2011

FIGURE 1: Recommendation of ESC guidelines 2011 on biomarkers for diagnosis and prognostic assessment in ACS (adapted from [8]).

therapy [38] were found to be largely restricted to patients with elevated cTn but in present those with normal $\mathrm{cTn}$ values. Regarding the need and timing of coronary angiography and coronary intervention, large randomized trials and a meta-analysis on 7 trials including 9,212 patients demonstrated that patients clearly derived benefits from an invasive strategy if a cardiac marker particularly cTn was elevated [39]. Although there is evidence that benefits from early invasive therapy and concomitant administration of GPIIb/IIIa inhibition may be achieved in patients with any detectable cTn level [37], inappropriate precision of conventional cTn assays precluded an unequivocal conclusion. Taking the lowest concentration that could be measured with appropriate precision showed a consistent benefit of triple antiplatelet therapy using aspirin, $600 \mathrm{mg}$ clopidogrel, and abciximab for ACS patients with cTn values exceeding $0.03 \mu \mathrm{g} / \mathrm{L}$, that is, the $10 \% \mathrm{CV}$ cutoff [38].

In contrast, the GUSTO IV trial found an excess of death among patients at low estimated risk characterized by a normal cTn and a NT-pro BNP below an optimal cutoff [32]. Whether patients with a normal hsTn will benefit from more potent antiplatelets or an early invasive strategy remained illusive until recently. In the PLATO trial [40] more than 18,000 patients with NSTE-ACS or STEMI were randomized to clopidogrel or ticagrelor and were allocated to an invasive or conservative strategy at the discretion of their physicians. Patients with hsTn levels below the 99th percentile value had a similar rate of the primary efficacy endpoint consisting of a composite of CV death, nonfatal MI, or stroke.

Other biomarkers, particularly markers of inflammation such as IL-6 have been investigated for their utility to guide the decision for an early invasive strategy [41]. The role of natriuretic peptides to guide invasive strategy has remained controversial due to conflicting study findings $[42,43]$. 


\section{References}

[1] J. Mackay, G. Mensah, S. Mendis, and K. Greenlund, The Atlas of Heart Disease and Stroke, World Health Organization, Geneva, Switzerland, 2004.

[2] H. A. Katus, A. Remppis, F. J. Neumann et al., "Diagnostic efficiency of troponin T measurements in acute myocardial infarction," Circulation, vol. 83, no. 3, pp. 902-912, 1991.

[3] H. A. Katus, A. Remppis, T. Scheffold, K. W. Diederich, and W. Kuebler, "Intracellular compartmentation of cardiac troponin T and its release kinetics in patients with reperfused and nonreperfused myocardial infarction," American Journal of Cardiology, vol. 67, no. 16, pp. 1360-1367, 1991.

[4] "Myocardial infarction redefined-a consensus document of The Joint European Society of Cardiology/American College of Cardiology Committee for the redefinition of myocardial infarction," Journal of the American College of Cardiology, vol. 36, no. 3, pp. 959-969, 2000.

[5] K. Thygesen, J. S. Alpert, A. S. Jaffe, M. L. Simoons, B. R. Chaitman, and H. D. White, "Third universal definition of myocardial infarction," Nature Reviews Cardiology, vol. 9, no. 11, pp. 620633, 2012.

[6] T. Reichlin, W. Hochholzer, S. Bassetti et al., "Early diagnosis of myocardial infarction with sensitive cardiac troponin assays," The New England Journal of Medicine, vol. 361, no. 9, pp. 858867, 2009.

[7] T. Keller, T. Zeller, D. Peetz et al., "Sensitive troponin I assay in early diagnosis of acute myocardial infarction," The New England Journal of Medicine, vol. 361, no. 9, pp. 868-877, 2009.

[8] C. W. Hamm, J. P. Bassand, S. Agewall et al., "ESC guidelines for the management of acute coronary syndromes in patients presenting without persistent ST-segment elevation: the Task Force for the management of acute coronary syndromes (ACS) in patients presenting without persistent ST-segment elevation of the European Society of Cardiology (ESC)," European Heart Journal, vol. 32, no. 23, pp. 2999-3054, 2011.

[9] E. Giannitsis and H. A. Katus, "Pros and cons of high-sensitivity assays for cardiac troponin," Nature Reviews. Cardiology, vol. 9, no. 11, pp. 616-618, 2012.

[10] K. Thygesen, J. Mair, H. Katus et al., "Recommendations for the use of cardiac troponin measurement in acute cardiac care," European Heart Journal, vol. 31, no. 18, pp. 2197-2204, 2010.

[11] E. Giannitsis, K. Kurz, K. Hallermayer, J. Jarausch, A. S. Jaffe, and H. A. Katus, "Analytical validation of a high-sensitivity cardiac troponin T assay," Clinical Chemistry, vol. 56, no. 2, pp. 254-261, 2010.

[12] A. K. Saenger, R. Beyrau, S. Braun et al., "Multicenter analytical evaluation of a high-sensitivity troponin T assay," Clinica Chimica Acta, vol. 412, no. 9-10, pp. 748-754, 2011.

[13] E. Giannitsis, M. Becker, K. Kurz, G. Hess, D. Zdunek, and H. A. Katus, "High-sensitivity cardiac troponin T for early prediction of evolving non-ST-segment elevation myocardial infarction in patients with suspected acute coronary syndrome and negative troponin results on admission," Clinical Chemistry, vol. 56, no. 4, pp. 642-650, 2010.

[14] K. Thygesen, J. Mair, E. Giannitsis et al., "How to use high-sensitivity cardiac troponins in acute cardiac care," European Heart Journal, vol. 33, no. 18, pp. 2252-2257, 2012.

[15] K. Kurz, E. Giannitsis, M. Becker, G. Hess, D. Zdunek, and H. A. Katus, "Comparison of the new high sensitive cardiac troponin $\mathrm{T}$ with myoglobin, h-FABP and cTnT for early identification of myocardial necrosis in the acute coronary syndrome," Clinical Research in Cardiology, vol. 100, no. 3, pp. 209-215, 2011.

[16] M. Than, L. Cullen, S. Aldous et al., "2-hour accelerated diagnostic protocol to assess patients with chest pain symptoms using contemporary troponins as the only biomarker: the ADAPT trial," Journal of the American College of Cardiology, vol. 59, no. 23, pp. 2091-2098, 2012.

[17] T. Reichlin, C. Schindler, B. Drexler et al., "One-hour rule-out and rule-in of acute myocardial infarction using high-sensitivity cardiac troponin T," Archives of Internal Medicine, vol. 172, no. 16, pp. 1211-1218, 2012.

[18] L. K. Newby, "Myocardial infarction rule-out in the emergency department: are high-sensitivity troponins the answer?: comment on 'One-hour rule-out and rule-in of acute myocardial infarction using high-sensitivity cardiac troponin T'” Archives of Internal Medicine, vol. 172, no. 16, pp. 1218-1219, 2012.

[19] T. Keller, S. Tzikas, T. Zeller et al., "Copeptin improves early diagnosis of acute myocardial infarction," Journal of the American College of Cardiology, vol. 55, no. 19, pp. 2096-2106, 2010.

[20] T. Reichlin, W. Hochholzer, C. Stelzig et al., "Incremental value of copeptin for rapid rule out of acute myocardial infarction," Journal of the American College of Cardiology, vol. 54, no. 1, pp. 60-68, 2009.

[21] E. Giannitsis, T. Kehayova, M. Vafaie, and H. A. Katus, "Combined testing of high-sensitivity troponin $\mathrm{T}$ and copeptin on presentation at prespecified cutoffs improves rapid rule-out of non-ST-segment elevation myocardial infarction," Clinical Chemistry, vol. 57, no. 10, pp. 1452-1455, 2011.

[22] M. Mueller, M. Biener, M. Vafaie et al., "Absolute and relative kinetic changes of high-sensitivity cardiac troponin $\mathrm{T}$ in acute coronary syndrome and in patients with increased troponin in the absence of acute coronary syndrome," Clinical Chemistry, vol. 58, no. 1, pp. 209-218, 2012.

[23] S. Agewall, E. Giannitsis, T. Jernberg, and H. Katus, "Troponin elevation in coronary vs. non-coronary disease," European Heart Journal, vol. 32, no. 4, pp. 404-411, 2011.

[24] K. M. Eggers, L. Lind, H. Ahlström et al., "Prevalence and pathophysiological mechanisms of elevated cardiac troponin I levels in a population-based sample of elderly subjects," European Heart Journal, vol. 29, no. 18, pp. 2252-2258, 2008.

[25] G. Korosoglou, S. Lehrke, D. Mueller et al., "Determinants of troponin release in patients with stable coronary artery disease: insights from CT angiography characteristics of atherosclerotic plaque," Heart, vol. 97, no. 10, pp. 823-831, 2011.

[26] A. Irfan, R. Twerenbold, M. Reiter et al., "Determinants of highsensitivity troponin $\mathrm{T}$ among patients with a noncardiac cause of chest pain," American Journal of Medicine, vol. 125, no. 5, pp. 491-498, 2012.

[27] S. Celik, E. Giannitsis, K. C. Wollert et al., "Cardiac troponin $\mathrm{T}$ concentrations above the 99th percentile value as measured by a new high-sensitivity assay predict long-term prognosis in patients with acute coronary syndromes undergoing routine early invasive strategy," Clinical Research in Cardiology, vol. 100, pp. 1077-1085, 2011.

[28] A. Filusch, E. Giannitsis, H. A. Katus, and F. J. Meyer, "Highsensitive troponin T: a novel biomarker for prognosis and disease severity in patients with pulmonary arterial hypertension," Clinical Science, vol. 119, no. 5, pp. 207-213, 2010.

[29] D. A. Pascual-Figal, T. Casas, J. Ordonez-Llanos et al., "Highly sensitive troponin $\mathrm{T}$ for risk stratification of acutely destabilized heart failure," American Heart Journal, vol. 163, no. 6, pp. 10021010, 2012. 
[30] G. J. Blake and P. M. Ridker, "C-reactive protein and other inflammatory risk markers in acute coronary syndromes," Journal of the American College of Cardiology, vol. 41, no. 4, supplement, pp. S37-S42, 2003.

[31] S. Q. Khan, R. J. O’Brien, J. Struck et al., "Prognostic value of midregional pro-adrenomedullin in patients with acute myocardial infarction: the LAMP (Leicester Acute Myocardial Infarction Peptide) study," Journal of the American College of Cardiology, vol. 49, no. 14, pp. 1525-1532, 2007.

[32] S. K. James, B. Lindahl, A. Siegbahn et al., "N-terminal probrain natriuretic peptide and other risk markers for the separate prediction of mortality and subsequent myocardial infarction in patients with unstable coronary artery disease: a Global Utilization of Strategies To Open occluded arteries (GUSTO)IV substudy," Circulation, vol. 108, no. 3, pp. 275-281, 2003.

[33] C. Meune, C. Balmelli, R. Twerenbold et al., "Utility of 14 novel biomarkers in patients with acute chest pain and undetectable levels of conventional cardiac troponin," International Journal of Cardiology, 2012.

[34] S. von Haehling, J. Papassotiriou, N. G. Morgenthaler et al., "Copeptin as a prognostic factor for major adverse cardiovascular events in patients with coronary artery disease," International Journal of Cardiology, vol. 162, no. 1, pp. 27-32, 2012.

[35] B. Lindahl, P. Venge, and L. Wallentin, “Troponin T identifies patients with unstable coronary artery disease who benefit from long-term antithrombotic protection. Fragmin in Unstable Coronary Artery Disease (FRISC) Study Group," Journal of the American College of Cardiology, vol. 29, no. 1, pp. 43-48, 1997.

[36] D. A. Morrow, E. M. Antman, M. Tanasijevic et al., "Cardiac troponin I for stratification of early outcomes and the efficacy of enoxaparin in unstable angina: a TIMI-11B substudy," Journal of the American College of Cardiology, vol. 36, no. 6, pp. 1812-1817, 2000.

[37] D. A. Morrow, C. P. Cannon, N. Rifai et al., "Ability of minor elevations of troponins I and $\mathrm{T}$ to predict benefit from an early invasive strategy in patients with unstable angina and nonST elevation myocardial infarction: results from a randomized trial," Journal of the American Medical Association, vol. 286, no. 19, pp. 2405-2412, 2001.

[38] A. Kastrati, J. Mehilli, F. J. Neumann et al., "Abciximab in patients with acute coronary syndromes undergoing percutaneous coronary intervention after clopidogrel pretreatment: the ISAR-REACT 2 randomized trial," Journal of the American Medical Association, vol. 295, no. 13, pp. 1531-1538, 2006.

[39] S. R. Mehta, C. P. Cannon, K. A. A. Fox et al., "Routine vs selective invasive strategies in patients with acute coronary syndromes: a collaborative meta-analysis of randomized trials," Journal of the American Medical Association, vol. 293, no. 23, pp. 2908-2917, 2005.

[40] L. Wallentin, R. C. Becker, A. Budaj et al., “Ticagrelor versus clopidogrel in patients with acute coronary syndromes," The New England Journal of Medicine, vol. 361, no. 11, pp. 1045-1057, 2009.

[41] T. Jernberg, B. Lindahl, A. Siegbahn et al., "N-terminal probrain natriuretic peptide in relation to inflammation, myocardial necrosis, and the effect of an invasive strategy in unstable coronary artery disease," Journal of the American College of Cardiology, vol. 42, no. 11, pp. 1909-1916, 2003.

[42] D. A. Morrow, J. A. de Lemos, M. S. Sabatine et al., "Evaluation of B-type natriuretic peptide for risk assessment in unstable angina/non-ST-elevation myocardial infarction: B-type natriuretic peptide and prognosis in TACTICS-TIMI 18," Journal of the American College of Cardiology, vol. 41, no. 8, pp. 1264-1272, 2003.

[43] S. K. James, J. Lindbäck, J. Tilly et al., "Troponin-T and N-terminal pro-B-type natriuretic peptide predict mortality benefit from coronary revascularization in acute coronary syndromes: a GUSTO-IV substudy," Journal of the American College of Cardiology, vol. 48, no. 6, pp. 1146-1154, 2006. 

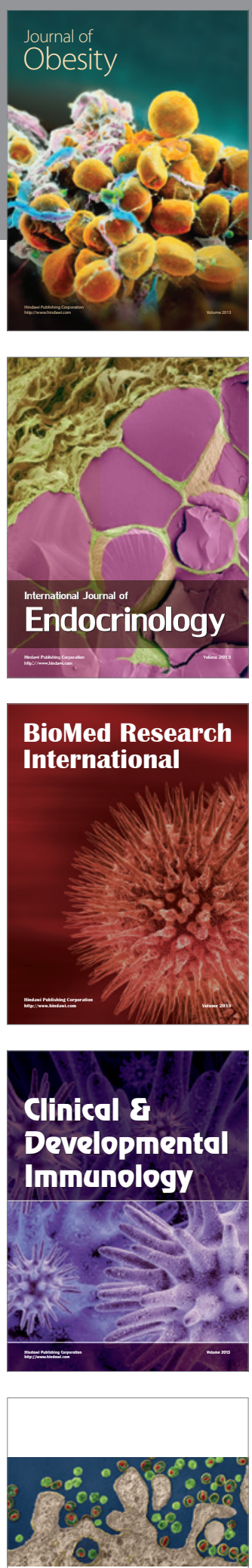

ISRN

AIDS
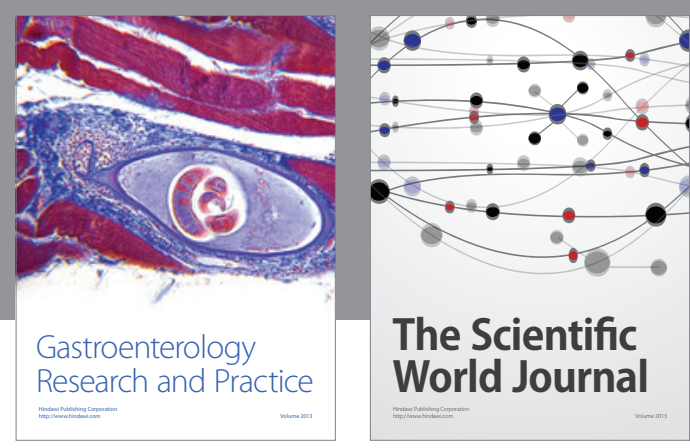

The Scientific World Journal
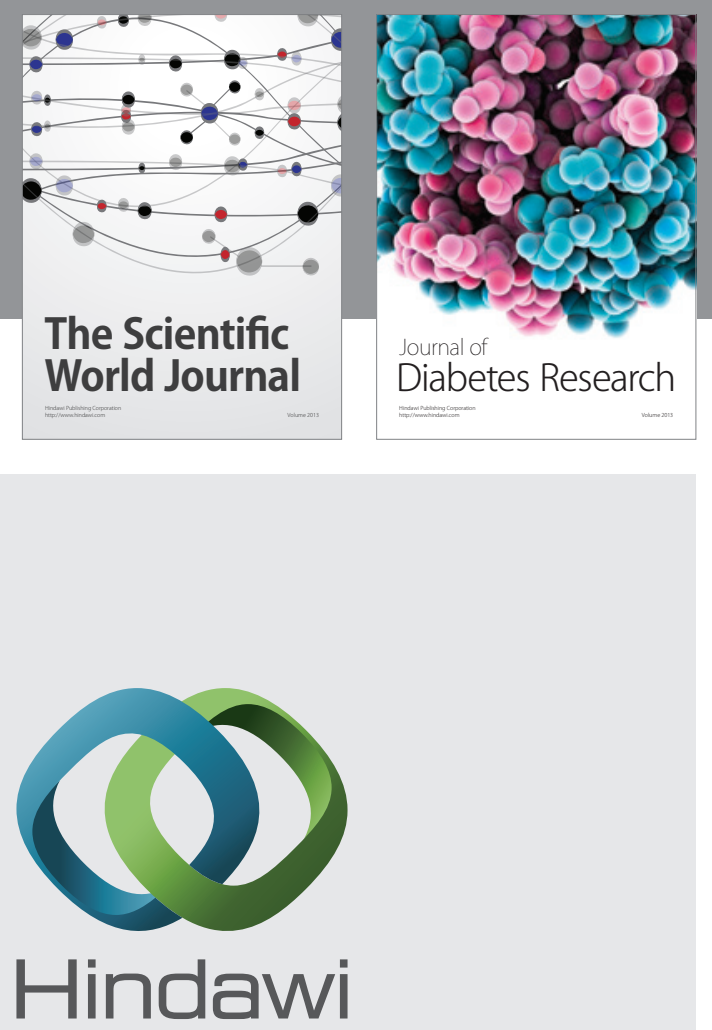

Submit your manuscripts at

http://www.hindawi.com
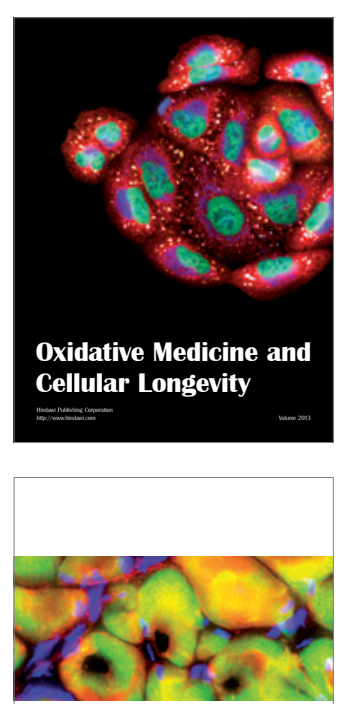

ISRN

Biomarkers
MEDIATORS

INFLAMMATION
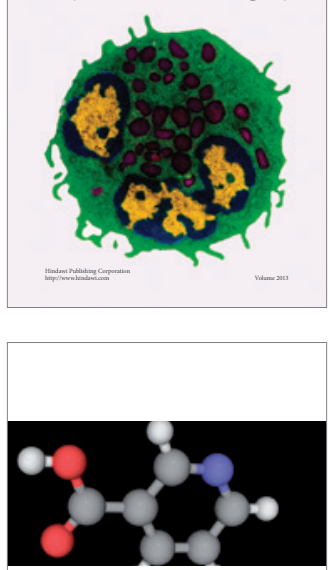

ISRN

Addiction
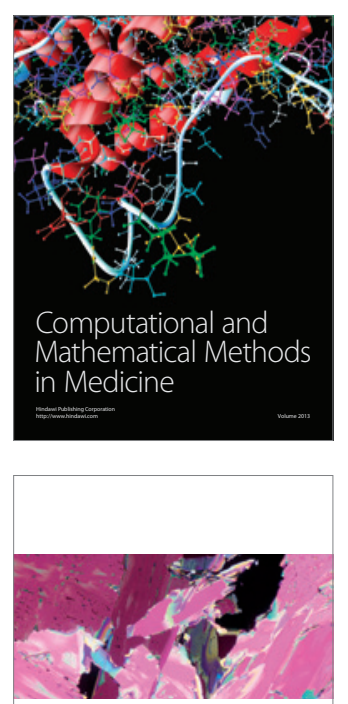

ISRN

Anesthesiology
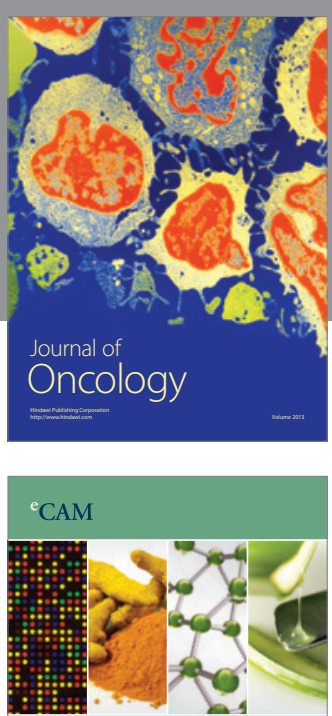

Evidence-Based

Complementary and

Alternative Medicine
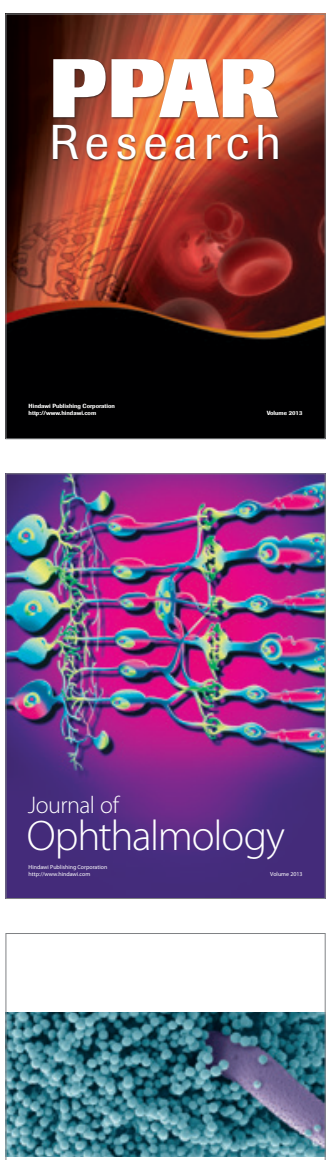

ISRN

Allergy 


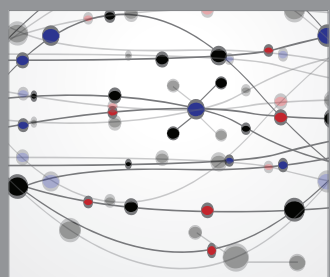

The Scientific World Journal
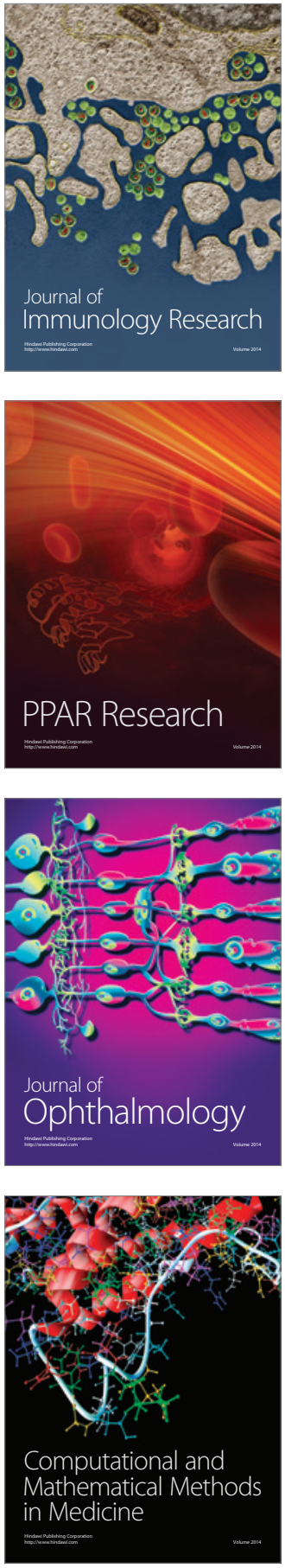

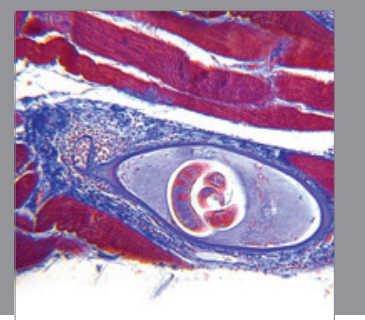

Gastroenterology

Research and Practice
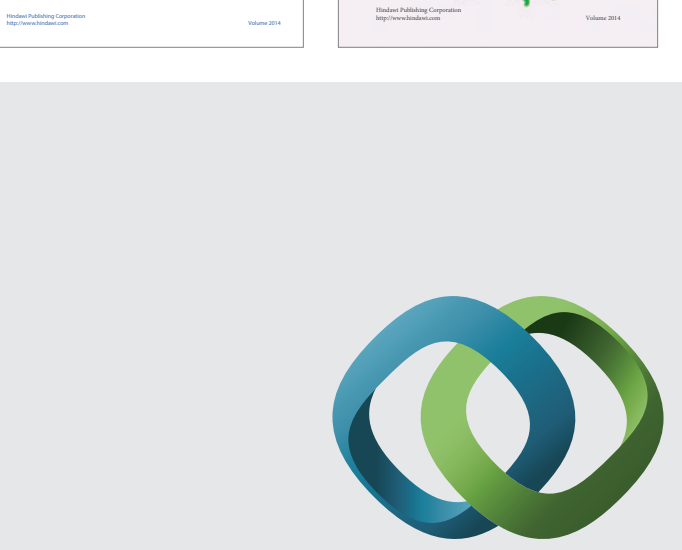

\section{Hindawi}

Submit your manuscripts at

http://www.hindawi.com
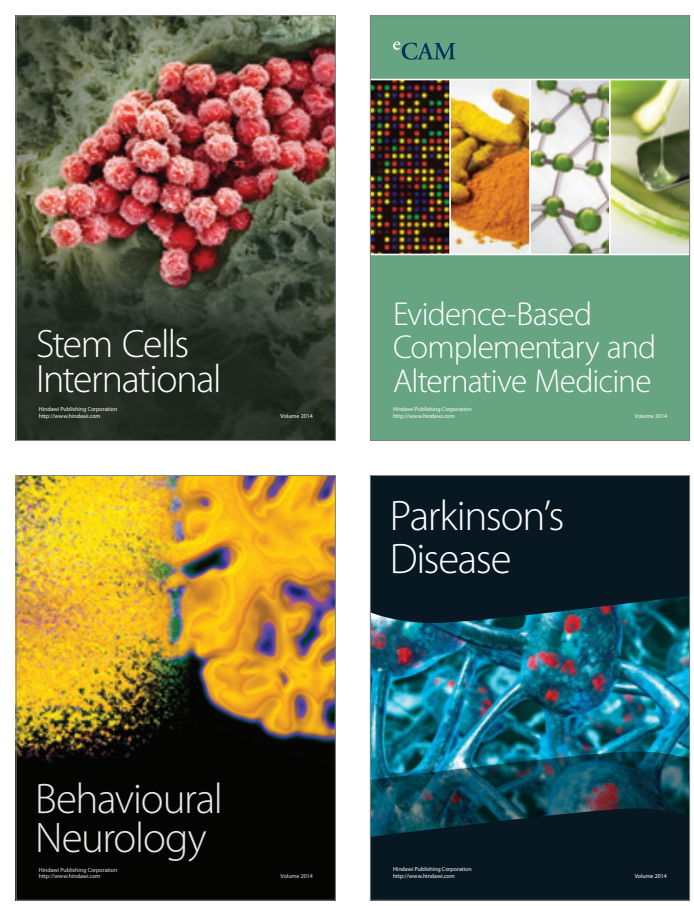

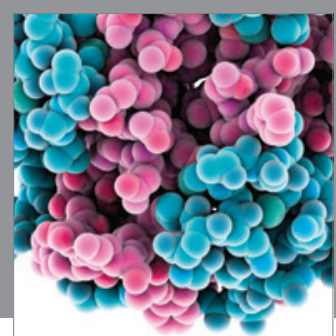

Journal of
Diabetes Research

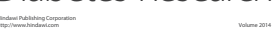

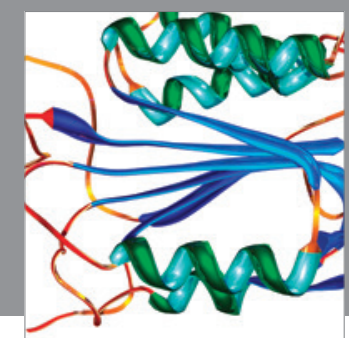

Disease Markers
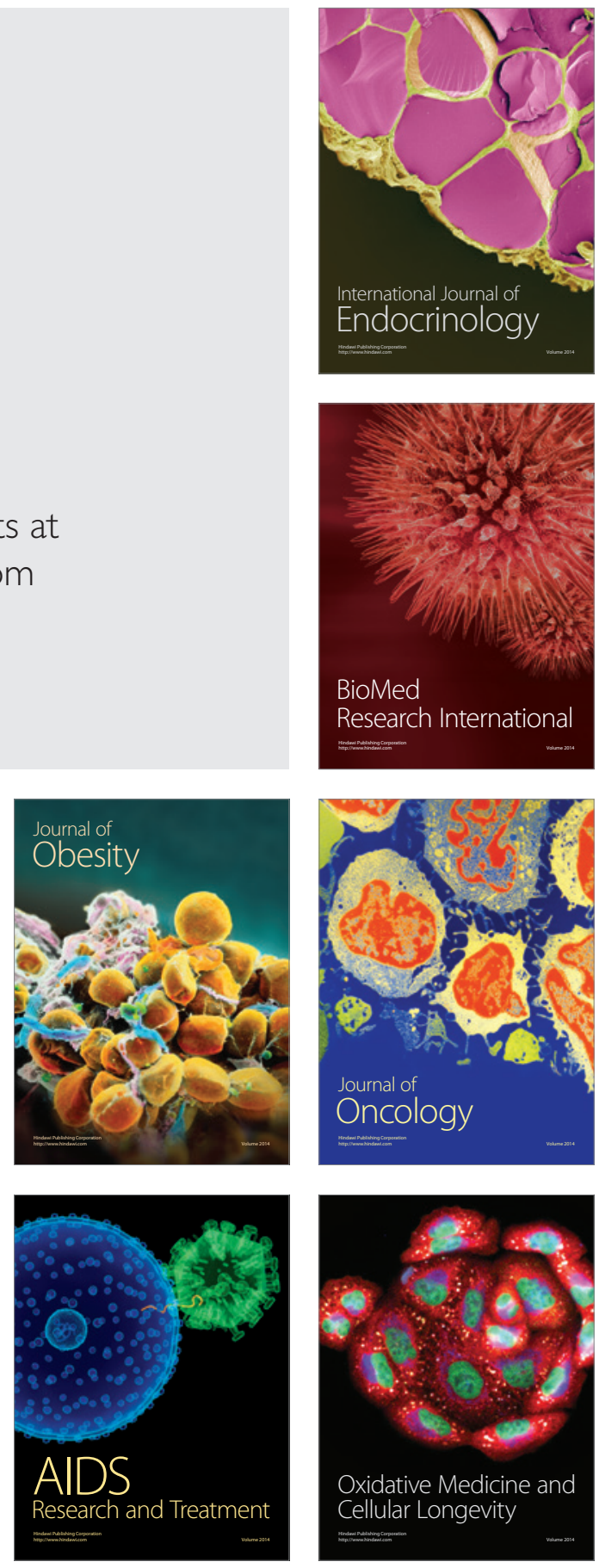